\title{
Effect of E-beam Dose on the Fractional Density of Au-Catalyzed GaAs Nanowire Growth
}

\author{
Jeung Hun Park ${ }^{\mathrm{a}, *}$, Vincent Gambin ${ }^{\mathrm{b}}$, Suneel Kodambaka ${ }^{\mathrm{a}, \dagger}$ \\ ${ }^{a}$ Department of Materials Science and Engineering, University of California Los Angeles, Los \\ Angeles, CA 90095, USA \\ ${ }^{b}$ Northrop Grumman Aerospace Systems, Redondo Beach, CA 90278, USA
}

\begin{abstract}
Using $\mathrm{Au} / \mathrm{GaAs}$ as a model system, the effect of initial catalyst conditions on nanowire densities was studied. Resulting morphologies and fractional surface densities are determined as a function of e-beam dose, dot size, and inter-dot spacing using scanning and transmission electron microscopies. The majority of resulting nanowires exhibited randomly oriented growth with the respect to the substrate and tapered with narrow tops, wider bases, and the catalyst tips characteristics of vapor-liquid-solid process. The base diameters of the wires are larger than the dot size, which is likely due to the non-catalyzed vapor-solid deposition along the sidewalls. The higher dose rate in pattering leads the formation of higher aspect ratio nanowires with narrower base. The fractional surface density follows the trend of clearing dose and critical dose for nanowire growth increases with decreasing catalyst pattering size and spacing. At a given dose, the fractional density increased with increasing Au dot size and with decreasing inter-dot spacing. Our results may provide new insights into the role of catalyst preparing conditions on the density controlled growth of nanowires in a single wafer.

\footnotetext{
*E-mail: jeunghunpark@gmail.com (Dr. J. H. Park)

†E-mail: kodambaka@ucla.edu (Prof. S. Kodambaka)

(C) 2016. This manuscript version is made available under the Elsevier user license http://www.elsevier.com/open-access/userlicense/1.0/
} 
Keywords: nanowire; vapor-liquid-solid process; catalyst conditions; e-beam lithography; molecular beam epitaxy; electron microscopy 


\section{Introduction}

Semiconducting group III-V nanowires, such as GaAs, InAs, and InP, owing to their high carrier mobilities and low band gaps, form an important class of materials with potential applications in nanoelectronics and optoelectronics, as chemical and biological sensors, and in energy harvesting devices [1,2]. To-date, nanowires have been grown using a variety of growth techniques such as self-catalyzed $[3,4,5]$, oxide-templated $[6,7,8]$, dislocation-assisted growth [9,10,11], among which the vapor-liquid-solid (VLS) [12] process is probably the most widely adapted method. And, considerable progress has been made over the past decade in developing methods to control the nanowire morphology, structure, and composition $[13,14,15,16]$. Waferscale growth of highly-ordered arrays of nanowires with nearly identical morphology, structure, and composition has been demonstrated using lithographically-patterned substrates as templates $[17,18,19,20,21]$. However, relatively little is known concerning the role of catalyst patterning parameters on the growth of nanowires. The properties of these nanowires were found to critically depend on the thickness and the linear dimension of the catalyst pattern elements.

In this paper, we report results from a detailed investigation of the influence of electronbeam dose during electron-beam lithography (EBL) on the morphology and the fractional surface density of nanowires. Au dots of two different sizes and three different inter-dot spacings are patterned via EBL on a 4" GaAs(111)B wafer. Several arrays of such patterns are prepared by applying a range of electron beam doses. In a single growth experiment, solid-source Ga and As precursors are used to grow GaAs nanowires on these patterned wafers. We studied the overall morphology and the fractional surface densities of the resulting nanowires as a function of electron beam dose, Au dot size, and inter-dot spacing. 


\section{Experimental Details}

All of our experiments are carried out on a 4" GaAs(111)B wafer. First, 200-nm-thick film of poly-methylmethacrylate (PMMA) is spin-coated on the wafer. Using EBL at $50 \mathrm{keV}$, we prepared 31 rows (labeled A to AE) of 11 identical patterns, as shown in Fig. 1. Each pattern consists of 6 rectangular regions that are made up of $250 \times 150$ ordered arrays of dots of nominal size $D(=100 \mathrm{~nm}$ and $150 \mathrm{~nm})$ separated by inter-dot spacing $S(=100 \mathrm{~nm}, 150 \mathrm{~nm}$, and $250 \mathrm{~nm})$. Each of the rows is patterned using a different electron beam dose $Q$ with increasing doses from $Q=145 \mu \mathrm{C} / \mathrm{cm}^{2}$ for the $\mathrm{A}^{\text {th }}$ row to $595 \mu \mathrm{C} / \mathrm{cm}^{2}$ for the $\mathrm{AE}^{\text {th }}$ row. The patterned wafer is developed at room temperature by dipping for $30 \mathrm{~s}$ in a liquid solvent composed of a mixture of isopropyl alcohol (IPA) and methyl isobutyl ketone (MIBK) with the ratio of 3:1. Au films, 1nm-thick, are then sputter-deposited on the patterned wafer and the wafer air-transferred to an ultra-high vacuum (UHV, base pressure $<5.5 \times 10^{-10}$ Torr) molecular beam epitaxy (MBE) system equipped with solid Ga and As sources for GaAs deposition. In the growth chamber, the wafer is cleaned of any surface oxides by heating at $480{ }^{\circ} \mathrm{C}$ for $600 \mathrm{~s}$ in the presence of As vapor maintained at a pressure of $1.05 \times 10^{-5}$ Torr. The sample is subsequently annealed at $485{ }^{\circ} \mathrm{C}$ for $\underline{120 \mathrm{~s}}$ in UHV to generate Au-Ga alloy droplets. Finally, nanowires are grown at $490{ }^{\circ} \mathrm{C}$ for 2400 s using a Ga beam pressure of $3.04 \times 10^{-7}$ Torr and a thermally cracked As 2 pressure of $1.05 \times$ $10^{-5}$ Torr. The as-grown samples are characterized using FEI Nova 600 field-emission scanning electron microscopy (SEM) and FEI Titan $300 \mathrm{keV}$ high resolution transmission electron microscopy (TEM). SEM images are processed using Image J software [22] to measure the image intensities of the patterns. From the SEM images of the dot patterns, we measured the dot sizes $D$ to be $106 \pm 5 \mathrm{~nm}$ and $144 \pm 5 \mathrm{~nm}$ and the inter-dot spacings $S$ to be $104 \pm 5 \mathrm{~nm}, 156 \pm 4$ $\mathrm{nm}$, and $258 \pm 4 \mathrm{~nm}$. Please note that in the following sections, we use the nominal $D(=100$ and 
$150 \mathrm{~nm})$ and $S(=100,150$, and $250 \mathrm{~nm})$ values for convenience. The fractional surface density of nanowires in a given pattern is determined [23]. In order to study the crystallinity of the GaAs nanowires, we obtained TEM images and diffraction patterns of individual nanowires which are mechanically exfoliated from the wafer, dispersed in ethanol and drop-casted onto a $3 \mathrm{~mm} \mathrm{Cu}$ grid with the holey carbon film.

\section{Results and Discussion}

Figure 2 shows representative secondary electron SEM images acquired from a thin $\mathrm{Au}$ covered GaAs(111)B sample with circular dot patterns created using EBL with $Q=$ (a) 220 $\mu \mathrm{C} / \mathrm{cm}^{2}$, (b) $295 \mu \mathrm{C} / \mathrm{cm}^{2}$, (c) $460 \mu \mathrm{C} / \mathrm{cm}^{2}$, and (d) $535 \mu \mathrm{C} / \mathrm{cm}^{2}$. In the patterns created using low electron beam doses, for example $Q=220 \mu \mathrm{C} / \mathrm{cm}^{2}$, the dots are barely visible. With increasing $Q$, we obtain well-defined dots with sharp boundaries. The plot in Fig. 2e shows spatial variations in normalized intensities of the SEM images in Figs. 2a-d. Here we define normalized intensity as the ratio of intensity at a given pixel with respect to average intensity of the image $[24,25]$. We find that the intensities of the dots increase with increasing $Q$. This trend is analogous to clearing dose in electron beam lithography where the amount of exposure energy required to just clear the resist in a large clear area for a given process increases linearly with e-beam dose $Q$ [26].

Figure 3a shows a typical SEM image of an individual GaAs nanowire grown in the $\mathrm{Y}^{\text {th }}$ row pattern $\left(D=150 \mathrm{~nm}, S=150 \mathrm{~nm}, Q=505 \mu \mathrm{C} / \mathrm{cm}^{2}\right)$. The nanowire is $2.5 \mu \mathrm{m}$ long and is strongly tapered with a base diameter of $\sim 192 \mathrm{~nm}$ and a tip diameter of $\sim 28 \mathrm{~nm}$. The top-view SEM image of the wire in Fig. $3 \mathrm{~b}$ shows that the wire is facetted with a hexagonal cross-section. Figures $3 \mathrm{c}-\mathrm{d}$ show top-view and $30^{\circ}$-tilted SEM images of vertically aligned GaAs nanowires grown in the $\mathrm{Z}^{\text {th }}$ row pattern $\left(D=150 \mathrm{~nm}, S=150 \mathrm{~nm}, Q=520 \mu \mathrm{C} / \mathrm{cm}^{2}\right)$. For this pattering 
condition, the majority of $\mathrm{Au}$ catalyzed GaAs nanowires exhibit epitaxial relationship with the respect to $\mathrm{GaAs}(111) \mathrm{B}$ substrate and significant tapering along the growth direction. The resulting morphology indicate that both vapor-liquid-solid and vapor-solid depositions are involved in the overall growth process [27,28].

From multiple SEM investigations, we measured the base diameter of individual nanowires. The obtained data was fitted to a log-normal distribution [29], and then their average diameters were calculated from the fitted slope. For $D \& S=100 \mathrm{~nm}$, the base diameter decreases from $253.3 \pm 2.7 \mathrm{~nm}$ to $167.9 \pm 2.6 \mathrm{~nm}$ as the electron beam dose increases from $Q=$

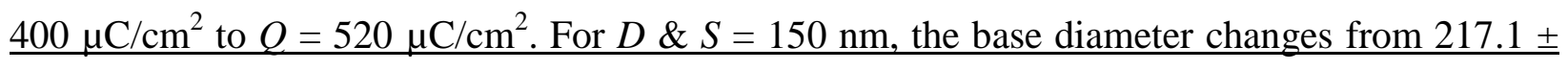

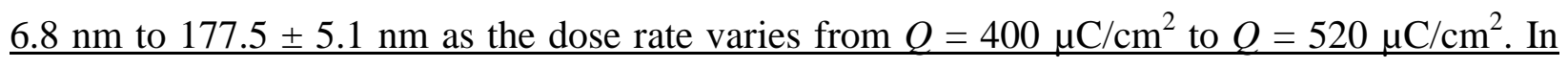
these dose ranges between $Q=400 \mu \mathrm{C} / \mathrm{cm}^{2}$ and $Q=520 \mu \mathrm{C} / \mathrm{cm}^{2}$, the average length of $\underline{\text { nanowires is } 3.0 \pm 0.5 \mu \mathrm{m} \text { but nanowires are observable up to } 10 \mu \mathrm{m} \text { in length. We observed that }}$ several nanowires grown from the same root within a circular dot pattern results in an irregular morphology and randomly oriented growth respect to the substrate. The shapes of nanowires are highly tapered along their length and with larger base diameters than the original pattern sizes i.e., $100 \mathrm{~nm}$ or $150 \mathrm{~nm}$. We also measured the average aspect ratio of nanowires. The aspect ratio is defined as the length of nanowire divided by the base diameter of the wire. For $D \& S=$ $100 \mathrm{~nm}$, the aspect ratio increases from 13.2 at $Q=400 \mu \mathrm{C} / \mathrm{cm}^{2}$ to 25.1 at $Q=520 \mu \mathrm{C} / \mathrm{cm}^{2}$. For $\underline{D \& S}=150 \mathrm{~nm}$, the ratio increases from 22.7 at $Q=400 \mu \mathrm{C} / \mathrm{cm}^{2}$ to 25.7 at $Q=520 \mu \mathrm{C} / \mathrm{cm}^{2}$. We found that the aspect ratio in the pattern $D \& S=100 \mathrm{~nm}$ is more sensitively changed to the dose rate than that in $D \& S=150 \mathrm{~nm}$. At the lower dose rate, the as-grown nanowires have lower aspect ratio structures with larger bases but higher aspect ratio structures with decreasingly 
narrow bases towards the higher dose rate. These results are also typical of all the nanowires grown in other patterns, irrespective of $D, S$, and $Q$.

Figure $4 \mathrm{a}$ is a bright-field TEM image of GaAs nanowire collected from the $\mathrm{Z}^{\text {th }}$ row pattern $\left(D=150 \mathrm{~nm}, S=150 \mathrm{~nm}, Q=520 \mu \mathrm{C} / \mathrm{cm}^{2}\right)$. The relatively uniform contrast along the wire is suggestive of spatially homogeneous structure and composition in the wire. As can be seen in Fig. 4a, the fact that the wire is highly tapered along with a smaller catalyst at the top is also indicative of uncatalzyed deposition of Ga and As species on the sides of the nanowires [28]. The inset in Fig. 4a is a selected area electron diffraction (SAED) pattern, viewed along the [112] axis, from the region highlighted in the TEM image. From the SAED data, we determined the crystal structure of nanowire as zinc blende (cubic) and the wire growth direction as $\langle 111\rangle[28]$. Figure $4 \mathrm{~b}$ shows the high resolution TEM image of the tip-wire interface. The darker contrast at the wire tip is likely due to the presence of an Au catalyst, expected for the vapor-solid-solid and $\underline{\text { VLS growth of nanowires [30,31]. From the lattice-resolved image, we measured inter-planar }}$ spacings of $0.23 \mathrm{~nm}$ and $0.33 \mathrm{~nm}$ for the tip and wire, respectively corresponding to $\langle 111\rangle$ planes in $\mathrm{Au}$ and GaAs [32]. We randomly selected 30 wires in other patterned regions and further analyzed TEM images. The catalyst tips are commonly observed in the wires (Figs. 4c-e). The overall length of nanowires was consisted with SEM analysis but rarely found up to $5 \mu \mathrm{m}$ (Fig. 4f). Irrespective of the electron beam patterning parameters $(D, S$, and $Q)$, the majority of nanowires contain a high density of planar defects (i.e. twins, stacking faults) and kinks along their growth direction (see Figs. $4 \mathrm{~g}$ and $\mathrm{h}$ ).

Overall nanowire morphology shows random orientation respect to substrate and broad $\underline{\text { size distribution of base diameter of nanowire, indicating none Au catalyzed nanowire growth }}$ that surface migration of Ga adatom with a mean length of $3 \sim 5 \mu \mathrm{m}$ may induce nanowire 
growth $[33,34,35]$. Random orientation of GaAs nanowires results in the large variations in $\mathrm{Au}-$ Ga catalyst [35,36], catalyst migration of $\mathrm{Au}[37,38]$ or $\mathrm{Ga}[39,40,41]$, and relatively lower growth temperature $[42,43,44]$. We note that the base diameters of the wires are larger than the Au dot size $D$, which is likely due to the non-catalyzed deposition along the sidewalls during the catalyst-assisted growth process [45]. It confirms that non-catalytic deposition (i.e. mainly for lateral growth) at the lower dose rate prevails VLS growth mechanism (i.e. primarily for vertical growth) $[5,27,46]$, in which the dose rate changes in the substrate pattering step may be used as one control parameter for adjusting the base diameter and aspect ratio within the constant growth $\underline{\text { conditions. }}$

In the following sections, we focus on understanding the effect of $D, S$, and $Q$ on the fractional surface density of nanowires. Figures 5a-d are top-view SEM images of the patterned areas created using different $Q$. Figure 5a is a low-magnification SEM image of the region containing two patterns $Q=340 \mu \mathrm{C} / \mathrm{cm}^{2}$ and $355 \mu \mathrm{C} / \mathrm{cm}^{2}$. The brighter contrast in the red rectangle is due to the presence of nanowires (see higher magnification image in Fig. 5b). The absence of any such contrast within the image is attributed to the lack of nanowires in that region. From a series of SEM images acquired from all the patterns, we find that regions patterned using $Q<355 \mu \mathrm{C} / \mathrm{cm}^{2}$ did not yield any nanowires. In Fig. 5b, we find nanowires in three out of the six patterns, which contain Au dots with $D=150 \mathrm{~nm}$ separated by distances $S=100 \mathrm{~nm}, 150 \mathrm{~nm}$, and $250 \mathrm{~nm}$ from left to right in the image. The other three patterns invisible in the image due to the lack of any nanowires contain smaller dots of size $D=100 \mathrm{~nm}$. We observed similar behavior in all the patterns prepared using doses $Q<400 \mu \mathrm{C} / \mathrm{cm}^{2}$. At higher doses, for example $Q=400 \mu \mathrm{C} / \mathrm{cm}^{2}$, nanowires are observed to grow in five out of the six patterns (see Fig. $5 \mathrm{c}$ ). And, at $Q>400 \mu \mathrm{C} / \mathrm{cm}^{2}$, nanowires are found in all the six patterns as shown in Fig. $5 \mathrm{~d}$. 
The effects of electron beam dose $Q$ and inter-dot spacing $S$ on the fractional surface density of nanowires grown using Au dots of size $D=100 \mathrm{~nm}$ are pictorially illustrated in Fig. 6 . For a given $D$ and $S$, the fractional density of nanowires in the patterns increases with increasing $Q$. The density is also found to increase with decreasing $S$. In our experiment, this behavior is consistently observed from all the patterns fabricated using a range of $Q$ values. From the images of the patterns, we measured the fractional surface density of nanowires as a function of $Q, D$, and $S$.

Figure 7 is a plot of the fractional surface density as a function of $Q$ for all the six different combinations of $D$ and $S$. Here we found that the fractional density changes are consistent with a clearing dose in which the residual thickness of the polymer film decreases with increasing dose $[47,48,49]$. For $Q \leq 350 \mu \mathrm{C} / \mathrm{cm}^{2}$, nanowires are not observed in any of the patterns, irrespective of $D$ and $S$. For $Q \geq 475 \mu \mathrm{C} / \mathrm{cm}^{2}$, nanowire yield is maximum (the nanowire density is $\sim 80 \%$ ) in all of the patterns and is independent of $Q$. In the patterns prepared using intermediate $Q$ values between $350 \mu \mathrm{C} / \mathrm{cm}^{2}$ and $475 \mu \mathrm{C} / \mathrm{cm}^{2}$, the nanowire coverage increases linearly with $Q$.

The observed effect of $Q$ can be understood as follows: at low electron beam doses, the pattern is underexposed and does not be completely developed. Consequently, $\mathrm{Au}$ is not deposited in the patterns and nanowires do not grow. At the intermediate electron beam dose but below the critical dose, only a fraction of the polymer chains in the PMMA film breaks up and the extent of damage increases linearly with $Q$ [26,47]. Therefore, some amount of $\mathrm{Au}$ is deposited in the patterns. While nanowires can grow out of these patterns, their base diameters are likely to be narrower and their lengths shorter owing to smaller sized catalysts at the tips which diffuse away during growth. A net effect is the reduced nanowire density in the patterns 
created using lower $Q$. Here we can define the critical dose $Q_{c}$; that is a minimum required dose for full development resist layer and fully saturated fractional density of nanowires. At electron beam doses above the critical dose $Q_{c}$ (i.e, $Q_{c} \approx 490 \mu \mathrm{C} / \mathrm{cm}^{2}$ for $D=100 \mathrm{~nm}$ and $S=250 \mathrm{~nm}$ ), the pattern film is completely developed. Hence, the amount of Au deposited and the nanowire yield are maximized in these patterns. We find that the critical dose $Q_{c}$ increases with increasing $S$ for a given $D$. This observation is consistent to $\underline{\mathrm{GaP}}$ and InAs nanowire growth $[17,50]$.

Interestingly, nanowire yield is also found to vary with the parameters $D$ and $S$. Figure 8 shows the effect of inter-dot spacing $S$ on the fractional surface density. This effect is more pronounced in the patterns obtained using intermediate $Q$ values, i.e. between $350 \mu \mathrm{C} / \mathrm{cm}^{2}$ and $475 \mu \mathrm{C} / \mathrm{cm}^{2}$. In Fig. 8, we find that the minimum dose $Q$ to yield nanowires is $\sim 350 \mu \mathrm{C} / \mathrm{cm}^{2}$ in the patterns with dots $D=150 \mathrm{~nm}$. In contrast, higher doses $\left(Q>380 \mu \mathrm{C} / \mathrm{cm}^{2}\right)$ are required to yield nanowires in the patterns with smaller dots $D=100 \mathrm{~nm}$. It is also shown that nanowire yield is higher in the patterns with smaller inter-dot spacing. For a given $Q$, smaller $S$ shows larger fractional density of nanowire growth.

In order to explain these results, we assume that a minimum volume of $\mathrm{Au}$, which may vary with growth temperature and flux, is necessary for the nucleation and growth of nanowires. During EBL, electron beam broadening and multiple scattering events can lead to the proximity effect $[47,51]$. This effect is likely to be more significant in the patterns with smaller $S$ and $D$. In Fig. 8, smaller dots require higher dosage than larger dots in satisfying the critical dose for the nucleation and growth of nanowires. Also, the proximity effect can limit on the minimum spacing between features [52]. The direct consequence of undesirable exposures next to small feature results in broadening its size of the small feature. Hence, the extent of damage to the polymer film around dots with small $S$ is slightly higher than dots with large $S$. At a given dose, 
those patterns with smaller $S$ will be developed into the larger pattern size than the intended size. In the $\mathrm{Au}$ deposition, those patterns may receive more amounts of $\mathrm{Au}$, which result in higher fractional density of nanowires.

\section{Conclusion}

We investigated the correlation between predeposited catalyst layers and nanowire density, varying electron beam dose, patterned dot size, and inter-dot spacing on the Aucatalyzed growth of GaAs nanowires using solid Ga and As sources on GaAs(111)B wafer. Using scanning and transmission electron microscopy, we determined the morphologies of asgrown nanowires and the fractional surface density of nanowires. Due to the characteristics of vapor-liquid-solid process for axial growth and vapor-solid process for radial growth, overall nanowire morphology showed random orientation respect to substrate and broad size distribution of base diameter of nanowire. At given Au dot size and spacing, the exposure of higher dose rate promotes the formation of higher aspect ratio nanowires with smaller bases. The fractional density of nanowires shows linear dependency on electron beam dosage and followed the trend of clearing dose in PMMA layer. At a given dose, the fractional density also increased with increasing $\mathrm{Au}$ dot size and with decreasing inter-dot spacing. Our results will be useful to identify the key parameters of electron beam lithography that are desirable for large-scale and density-controlled growth of nanowires in a single wafer.

\section{Acknowledgements}

The authors would like to thank Drs. S. Prikhodko and M. Pozuelo for their helps in transmission electron microscopic characterization of GaAs nanowires. We gratefully acknowledge financial 
support from the UC Discovery - Northrop Grumman Aerospace Systems and the NSF though the grant CMMI-0926412. 


\section{FIGURE CAPTIONS}

Fig. 1. Schematic overview of patterns on $\operatorname{GaAs}(111) \mathrm{B}$ wafer. Each of the 31 patterns labeled alphabetically from A to AE consists of 6 rectangular regions made up of $250 \times 150$ ordered arrays of a given dot-size $D(=100$ and $150 \mathrm{~nm})$ and inter-dot spacing $S(=100,150$, and 250 $\mathrm{nm})$.

Fig. 2. Effect of electron beam dose $Q$ on the patterned dot profiles. Top-view field-emission SEM images acquired from a GaAs(111)B wafer patterned using EBL with dots of size $D=100$ $\mathrm{nm}$ and inter-dot spacing $S=150 \mathrm{~nm}$ as a function of $Q=$ (a) $220 \mu \mathrm{C} / \mathrm{cm}^{2}$, (b) $295 \mu \mathrm{C} / \mathrm{cm}^{2}$, (c) $460 \mu \mathrm{C} / \mathrm{cm}^{2}$, and (d) $535 \mu \mathrm{C} / \mathrm{cm}^{2}$. Scale bar in all the images is $500 \mathrm{~nm}$. (e) Spatial variations in the SEM image intensities of the patterns in (a)-(d) normalized with respect to the back ground intensity. Each of the curves is an average of over 128 line profiles acquired from the patterned regions.

Fig. 3. (a) $30^{\circ}$-tilted SEM image of a GaAs nanowire grown in the area containing $\mathrm{Au}$ dots of

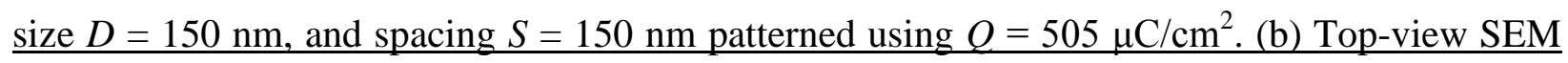
image of the same nanowire $\left(D=150 \mathrm{~nm}, S=150 \mathrm{~nm}, Q=505 \mu \mathrm{C} / \mathrm{cm}^{2}\right)$. (c) Top-view SEM image of GaAs nanowire arrays grown in the area containing Au dots of size $D=150 \mathrm{~nm}$, and spacing $S=150 \mathrm{~nm}$ patterned using $Q=520 \mu \mathrm{C} / \mathrm{cm}^{2}$. (d) $30^{\circ}$-tilted SEM image of GaAs nanowire arrays $\left(D=150 \mathrm{~nm}, S=150 \mathrm{~nm}, Q=520 \mu \mathrm{C} / \mathrm{cm}^{2}\right)$. 
Fig. 4. (a) Bright-field TEM image of a GaAs nanowire grown in the area prepared using $Q=$

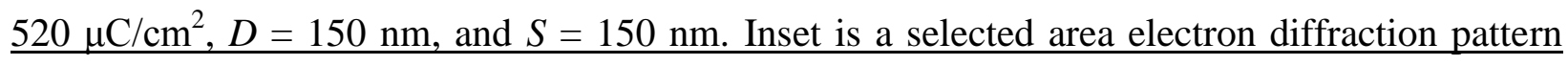
acquired along the $[112]$ zone axis from the region highlighted in the image. The diffraction data indicates that the wire is zinc blende in structure and the growth direction is $\langle 111\rangle$. (b) Higher resolution TEM images of the catalyst tip-wire interface. (c-e) HR-TEM images of the catalyst tips in the GaAs nanowires which are indicative of the characteristics of vapor-liquid-solid growth mechanism. All scale bars are $10 \mathrm{~nm}$. (f-g) Bright-field TEM image of GaAs nanowire obtained from randomly selected wires at the various dose rates $Q$ for $D=150 \mathrm{~nm}$ and $S=150$ nm. Significant tapering of nanowires indicated the growth of individual nanowires occurs via both vapor-liquid-solid and vapor-solid growth process. (h) Bright-field TEM image of GaAs nanowire obtained from the pattering condition of $Q=520 \mu \mathrm{C} / \mathrm{cm}^{2}, D=150 \mathrm{~nm}$, and $S=150 \mathrm{~nm}$. The SAED pattern indicates the growth direction of the wire is $\langle 111\rangle$.

Fig. 5. Top-view SEM image obtained from the patterned GaAs(111)B wafer after the growth of GaAs nanowires. a) Low-magnification SEM image of the region containing the pattern prepared using $Q=355 \mu \mathrm{C} / \mathrm{cm}^{2}$, highlighted by red square. The scale bar is $0.5 \mathrm{~mm}$. (b) Higher magnification SEM image of the red rectangular region in (a). (c,d) SEM images of the areas

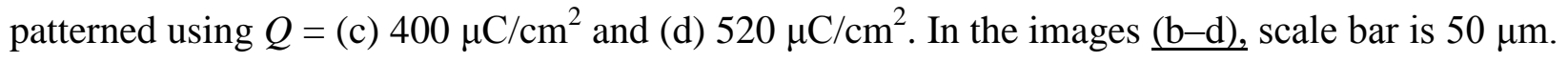
All the dots in a given row have the same size $D=150 \mathrm{~nm}$ (top row) and $100 \mathrm{~nm}$ (bottom row), while $S$ is $100 \mathrm{~nm}, 150$, and $250 \mathrm{~nm}$ in the left, center, and the right patterns, respectively. 
Fig. 6. SEM images of a GaAs(111)B wafer with GaAs nanowires grown in the areas patterned with Au dots of size $D=100 \mathrm{~nm}$. The vertical and horizontal axes show patterns obtained using different $Q$ and $S$ values, respectively. Scale bars in the images are $4 \mu \mathrm{m}$.

Fig. 7. The fractional surface density of nanowires plotted as a function of $Q$. Solid lines are a guide for the eye. The critical dose $Q_{c}$ is a minimum required dose for fully saturated fractional density of nanowires.

Fig. 8. The fractional surface density of nanowires plotted as a function of $S$ for (a) $D=100 \mathrm{~nm}$ and (b) $D=150 \mathrm{~nm}$ with four different e-beam doses $Q=355,400,430$, and $520 \mu \mathrm{C} / \mathrm{cm}^{2}$. Solid lines are a guide for the eye. 


\section{References}

[1] L. Chen, W. Lu, C.M. Lieber, Chapter 1: Semiconductor Nanowire Growth and Integration, in Semiconductor Nanowires: From Next-Generation Electronics to Sustainable Energy, 2014, pp. 1-53. DOI: $10.1039 / 9781782625209-00001$

[2] X. Zhuang, C.Z. Ning, A. Pan, Composition and bandgap-graded semiconductor alloy nanowires, Adv. Mater. 24 (2012) 13-33.

[3] L. Gao, R.L. Woo, B. Liang, M. Pozuelo, S. Prikhodko, M. Jackson, N. Goel, M.K. Hudait, D.L. Huffaker, M.S. Goorsky, S. Kodambaka, R.F. Hicks, Self-catalyzed epitaxial growth of vertical indium phosphide nanowires on silicon, Nano Lett. 9 (2009) 2223-2228.

[4] K.A. Dick, Philippe Caroff, Metal-seeded growth of III-V semiconductor nanowires: towards gold-free synthesis, Nanoscale 6 (2014) 3006-3021.

[5] S. Ermez, E.J. Jones, S.C. Crawford, S. Gradečak, Self-seeded growth of GaAs nanowires by metal-organic chemical vapor deposition, Cryst. Growth Des. 15 (2015) 2768-2774.

[6] Z.H. Wu, X.Y. Mei, D. Kim, M. Blumin, H.E. Ruda, Growth of Au-catalyzed ordered GaAs nanowire arrays by molecular-beam epitaxy, Appl. Phys. Lett. 81 (2002) 5177-5179.

[7] T.V. Hakkarainen, A. Schramm, J. Mäkelä, P. Laukkanen, M. Guina, Lithography-free oxide patterns as templates for self-catalyzed growth of highly uniform GaAs nanowires on $\mathrm{Si}(111)$, Nanotechnology 26 (2015) 275301.

[8] M.T. Robson, V.G. Dubrovskii, R.R. LaPierre, Conditions for high yield of selective-area epitaxy InAs nanowires on $\mathrm{SiO}_{\underline{x}} / \mathrm{Si}(111)$ substrates, Nanotechnology 26 (2015) 465301.

[9] M.J. Bierman, Y.K.A. Lau, A.V. Kvit, A.L. Schmitt, S. Jin, Dislocation-driven nanowire growth and Eshelby twist, Science 320 (2008) 1060-1063. 
[10] H. Wu, F. Meng, L. Li, S. Jin, G. Zheng, Dislocation-driven CdS and CdSe nanowire growth, ACS Nano 6 (2012) 4461-4468.

[11] F. Meng, S.A. Morin, A. Forticaux, S. Jin, Screw dislocation driven growth of nanomaterials, Acc. Chem. Res.46 (2013) 1616-1626.

[12] R.S. Wagner, W.C. Ellis, Vapor-liquid-solid mechanism of single crystal growth, Appl. Phys. Lett. 4 (1964) 89-90.

[13] Z. Fan, J.C. Ho, Z.A. Jacobson, R. Yerushalmi, R.L. Alley, H. Razavi, A. Javey, Waferscale assembly of highly ordered semiconductor nanowire arrays by contact printing, Nano Lett. 8 (2008) 20-25.

[14] F. Panciera, Y.-C. Chou, M.C. Reuter, D. Zakharov, E.A. Stach, S. Hofmann, F.M. Ross, $\underline{\text { Synthesis of nanostructures in nanowires using sequential catalyst reactions, Nat. Mater. } 14}$ (2015) 820-825.

[15] Y.-C. Chou, K. Hillerich, J. Tersoff, M.C. Reuter, K.A. Dick, F. M. Ross, Atomic-scale variability and control of III-V nanowire growth kinetics. Science 343 (2014) 281-284. [16] W. Du, X. Yang, H. Pan, X. Ji, H. Ji, S. Luo, X. Zhang, Z. Wang, T. Yang, Controlleddirection growth of planar InAsSb nanowires on Si substrates without foreign catalysts, Nano Lett. ASAP (2016). DOI: 10.1021/acs.nanolett.5b03587

[17] M.T. Borgström, G. Immink, B. Ketelaars, R. Algra, E.P.A.M. Bakkers, Synergetic nanowire growth, Nat. Nanotech. 2 (2007) 541-544.

[18] Y. Wei, W. Wu, R. Guo, D. Yuan, S. Das, Z.L. Wang, Wafer-scale high-throughput ordered growth of vertically aligned ZnO nanowire arrays, Nano Lett. 10 (2010) 3414-3419. 
[19] A.R. Madaria, M. Yao, C.Y. Chi, N. Huang, C. Lin, R. Li, M.L. Povinelli, P.D. Dapkus, C. Zhou, Toward optimized light utilization in nanowire arrays using scalable nanosphere lithography and selected area growth, Nano Lett. 12 (2013) 2839-2845. [20] J. Yao, H. Yan, C.M. Lieber, A nanoscale combing technique for the large-scale assembly of highly aligned nanowires, Nat. Nanotechnol. 8 (2013) 329-335.

[21] S.M. Nicaise, J.J. Cheng, A. Kiani, S. Gradečak, K.K. Berggren, Control of zinc oxide $\underline{\text { nanowire array properties with electron-beam lithography templating for photovoltaic }}$ applications, Nanotechnology 26 (2015) 075303. [22] C.A. Schneider, W.S. Rasband, K.W. Eliceiri, NIH Image to ImageJ: 25 years of image analysis, Nat. Methods 9 (2012) 671-675. [23] Y. Du, S. Han, W. Jin, C. Zhou, A.F.J. Levi, Polarization-dependent reflectivity from dielectric nanowires, Appl. Phys. Lett. 83 (2003) 996-998.

[24] S. Babin, K. Bay, J.J. Hwu, Application of analytic scanning electron microscopy to critical dimensions metrology at nanometer scale, J. Vac. Sci. Technol. B 28 (2010) C6H1. [25] Y. Kita, Y. Kasai, S. Hashimoto, K. Iiyama, S. Takamiya, Application of brightness of scanning electron microscope images to measuring thickness of nanometer-thin $\mathrm{SiO}_{2}$ layers on $\mathrm{Si}$ substrates, Jpn. J. Appl. Phys. 40 (2001) 5861-5864.

[26] S. Yasin, M.N. Khalid, D.G. Hasko, S. Sarfraz, Correlation of surface roughness with edge roughness in PMMA resist, Microelectron. Eng. 78/79 (2005) 484-489.

[27] S. Krylyuk, A. V. Davydov, I. Levin, Tapering control of Si nanowires grown from SiCl4 at reduced pressure, ACS Nano 5 (2011) 656-664. 
[28] N. Jiang, J. Wong-Leung, H.J. Joyce, Q. Gao, H.H. Tan, C. Jagadish, Understanding the true shape of au-catalyzed GaAs nanowires, Nano Lett. 14 (2014) 5865-5872.

[29] Q. Xiong, R. Gupta, K.W. Adu., E.C. Dickey, G.D. Lian, D. Tham, J.E. Fischer, P.C. Eklund, Raman spectroscopy and structure of crystalline gallium phosphide nanowires, J. Nanosci. Nanotech. 3 (2003) 335-339.

[30] S. Kodambaka, J. Tersoff, M.C. Reuter, F.M. Ross, Germanium nanowire growth below the eutectic temperature, Science 316 (2007) 729-732.

[31] A.I. Persson, M.W. Larsson, S. Stenström, B.J. Ohlsson, L. Samuelson, L.R. Wallenberg, Solid-phase diffusion mechanism for GaAs nanowire growth, Nat. Mater. 3 (2004) 677-681. [32] ICDD-JCPDS Catalogue No. 32-0389.

[33] J.C. Harmand, G. Patriarche, N. Péré-Laperne, M-N. Mérat-Combes, L. Travers, F. Glas, Analysis of vapor-liquid-solid mechanism in Au-assisted GaAs nanowire growth, Appl. Phys. Lett. 87 (2005) 203101.

[34] Z-M. Liao, Z-G. Chen, Z-Y. Yu, H-Y. Xi, Y-N. Guo, W. Sun, Z. Zhang, L. Yang, P-P. Chen, W. Lu, J. Zou, Au impact on GaAs epitaxial growth on GaAs (111)B substrates in molecular beam epitaxy, Appl. Phys. Lett. 102 (2013) 063106.

[35] H.J. Joyce, Q. Gao, H.H. Tan, C. Jagadish, Y. Kim, M.A. Fickenscher, S. Perera, T.B. Hoang, L.M. Smith, H.E. Jackson, J.M. Yarrison-Rice, X. Zhang, J. Zou, High purity GaAs nanowires free of planar defects: growth and characterization, Adv. Funct. Mater. 18 (2008) 3794-3800. 
[36] S.O. Mariager, S.L. Lauridsen, C.B. Sørensen, A. Dohn, P.R. Willmott, J. Nygård, R. Feidenhans'1, Stages in molecular beam epitaxy growth of GaAs nanowires studied by x-ray diffraction, Nanotechnology 21 (2010) 115603.

[37] J.B. Hannon, S. Kodambaka, F.M. Ross, R.M. Tromp, The influence of the surface migration of gold on the growth of silicon nanowires, Nature 440 (2006) 69-71. [38] A.A. Bonapasta, F. Buda, Binding and migration paths of Au adatoms on the GaAs(001) surface, Phys. Rev. B 65 (2002) 045308.

[39] J. Tersoff, D.E. Jesson, W.X. Tang, Running droplets of gallium from evaporation of gallium arsenide, Science 324 (2009) 236-238.

[40] T.A. Bryantseva, D.V. Lioubtchenko, V.V. Lopatin, Ga migration process in Au film on (100) GaAs under temperature treatment in vacuum, Appl. Surf. Sci. 100/101 (1996) 169-173. [41] S. Kodambaka, C. Ngo, J. Palisaitis, P.H. Mayrhofer, L. Hultman, P.O.Å. Persson, Kinetics of Ga droplet decay on thin carbon films, Appl. Phys. Lett. 102 (2013) 161601.

[42] P. Mohan, R. Bag, S. Singh, A. Kumar, R. Tyagi, Mechanism of self-assembled growth of ordered GaAs nanowire arrays by metalorganic vapor phase epitaxy on GaAs vicinal substrates, Nanotechnology 23 (2012) 025601.

[43] M. Tchernycheva, J.C. Harmand, G. Patriarche, L. Travers, G.E. Cirlin, Temperature conditions for GaAs nanowire formation by Au-assisted molecular beam epitaxy, Nanotechnology 17 (2006) 4025-4030.

[44] V.G. Dubrovskii, M.A. Timofeeva, A. Kelrich, D. Ritter, Growth and morphological modeling of InP nanowires obtained by Au-catalyzed selective area MOMBE, J. Cryst. Growth $413(2015) 25-30$. 
[45] V.G. Dubrovskii, I.P. Soshnikov, G.E. Cirlin, A.A. Tonkikh, Y.B. Samsonenko, N.V.

Sibirev, V.M. Ustinov, On the non-monotonic lateral size dependence of the height of GaAs nanowhiskers grown by molecular beam epitaxy at high temperature, Phys. Status Solidi B 241 (2004) R30-R33.

[46] R.L. Woo, L. Gao, N. Goel, M.K. Hudait, K.L. Wang, S. Kodambaka, R.F. Hicks, Kinetic control of self-catalyzed indium phosphide nanowires, nanocones, and nanopillars, Nano Lett. 9 (2009) 2207-2211.

[47] J. Kretz, L. Dreeskornfeld, G. Ilicali, T. Lutz, W. Weber, Comparative study of calixarene and HSQ resist systems for the fabrication of sub-20 nm MOSFET device demonstrators, Microelectron. Eng. 78/79 (2005) 479-483.

[48] F.P. Gibbons, J. Manyam, S. Diegoli, M. Manickam, J.A. Preece, R.E. Palmer, A.P.G. Robinson, Chemically amplified molecular resists for e-beam lithography, Microelectron. Eng. 85 (2008) 764-767.

[49] A.E. Grigorescu, C.W. Hagen, Resists for sub-20-nm electron beam lithography with a focus on HSQ: state of the art, Nanotechnology 20 (2009) 292001.

[50] S. Hertenberger, D. Rudolph, M. Bichler, J. J. Finley, G. Abstreiter, G. Koblmüller, Growth kinetics in position-controlled and catalyst-free InAs nanowire arrays on $\mathrm{Si}(111)$ grown by $\underline{\text { selective area molecular beam epitaxy, J. Appl. Phys. } 108 \text { (2010) } 114316 .}$ [51] T.H.P. Chang, Proximity effect in electron-beam lithography, J. Vac. Sci. Technol. 12 (1975) $1271-1275$. 
[52] X. Zhang, Z. Fang, K. Chen, J. Xu, X. Huang, Fabricating a silicon nanowire by using the proximity effect in electron beam lithography for investigation of the Coulomb blockade effect, Nanotechnology 22 (2011) 035302. 


\section{Figure}

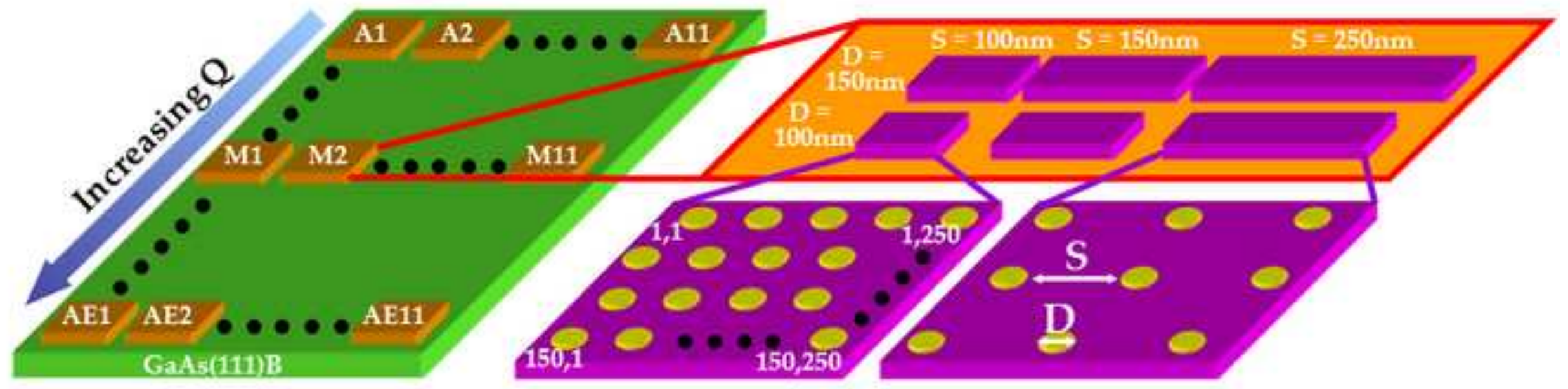




\section{(a) $220 \mu \mathrm{C} / \mathrm{cm}^{2}$}

\section{(b) $295 \mu \mathrm{C} / \mathrm{cm}^{2}$}

$500 \mathrm{~nm}$

(d) $535 \mu \mathrm{C} / \mathrm{cm}^{2}$
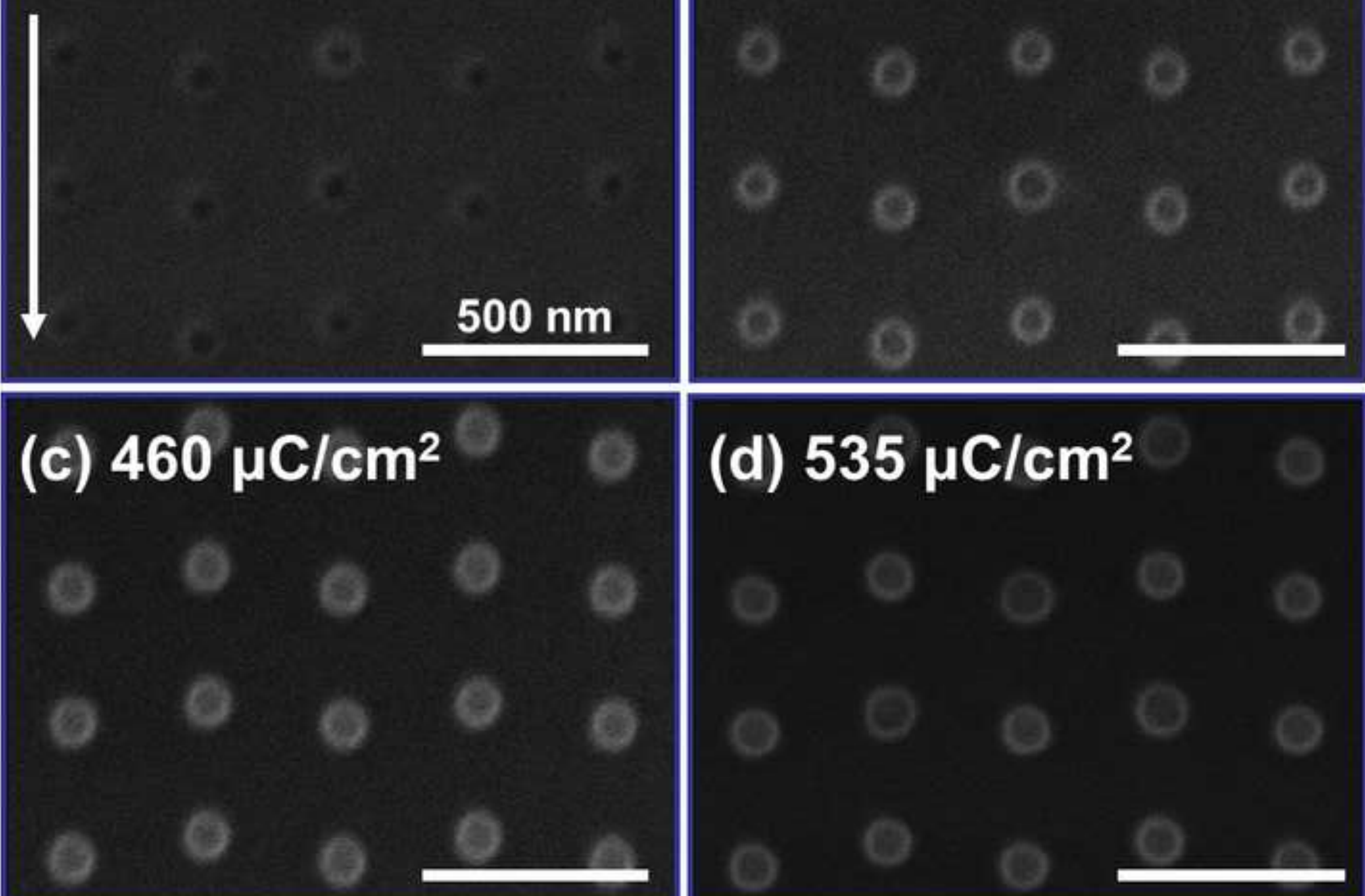

\section{(c) $460 \mu \mathrm{C} / \mathrm{cm}^{2}$}

0




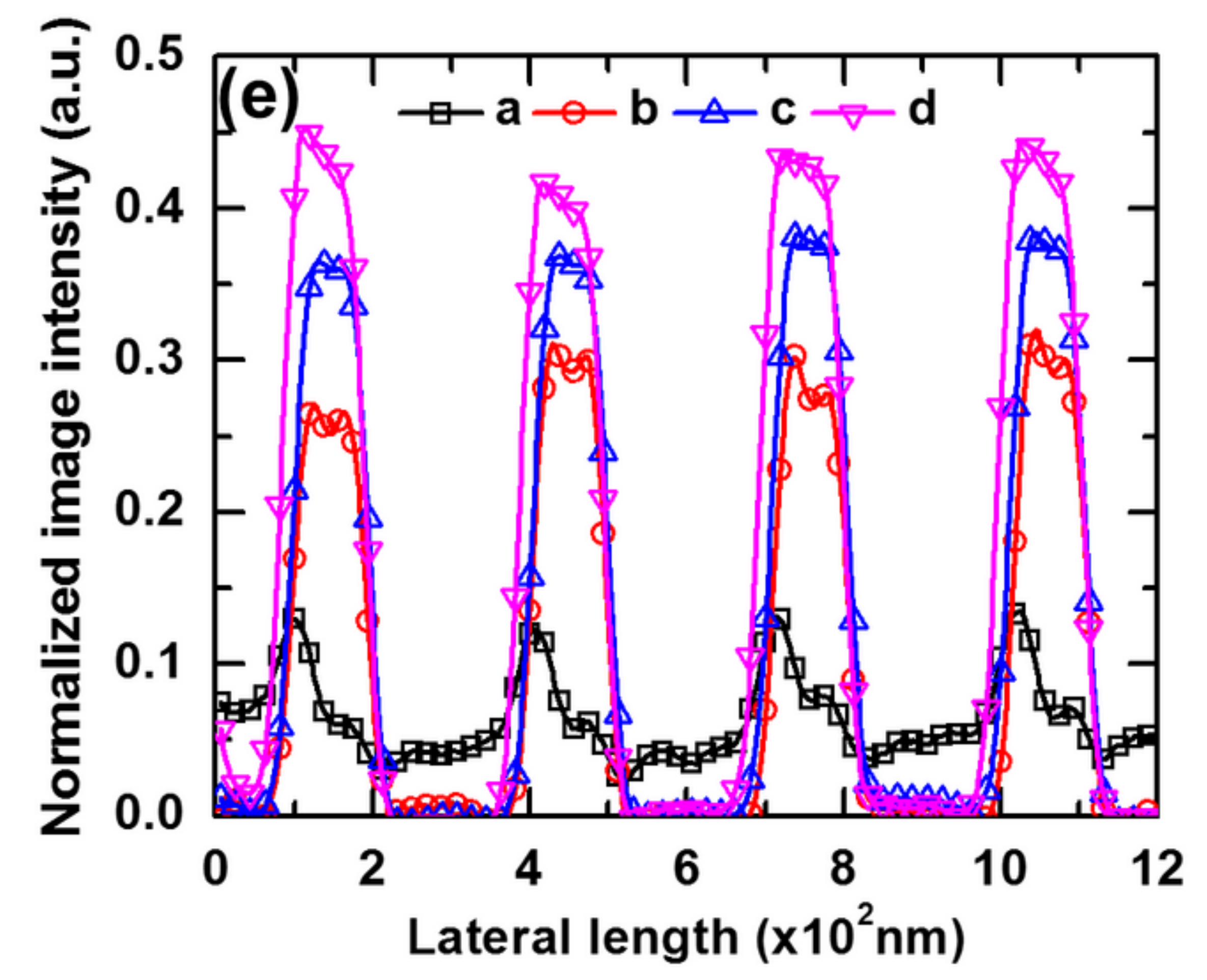




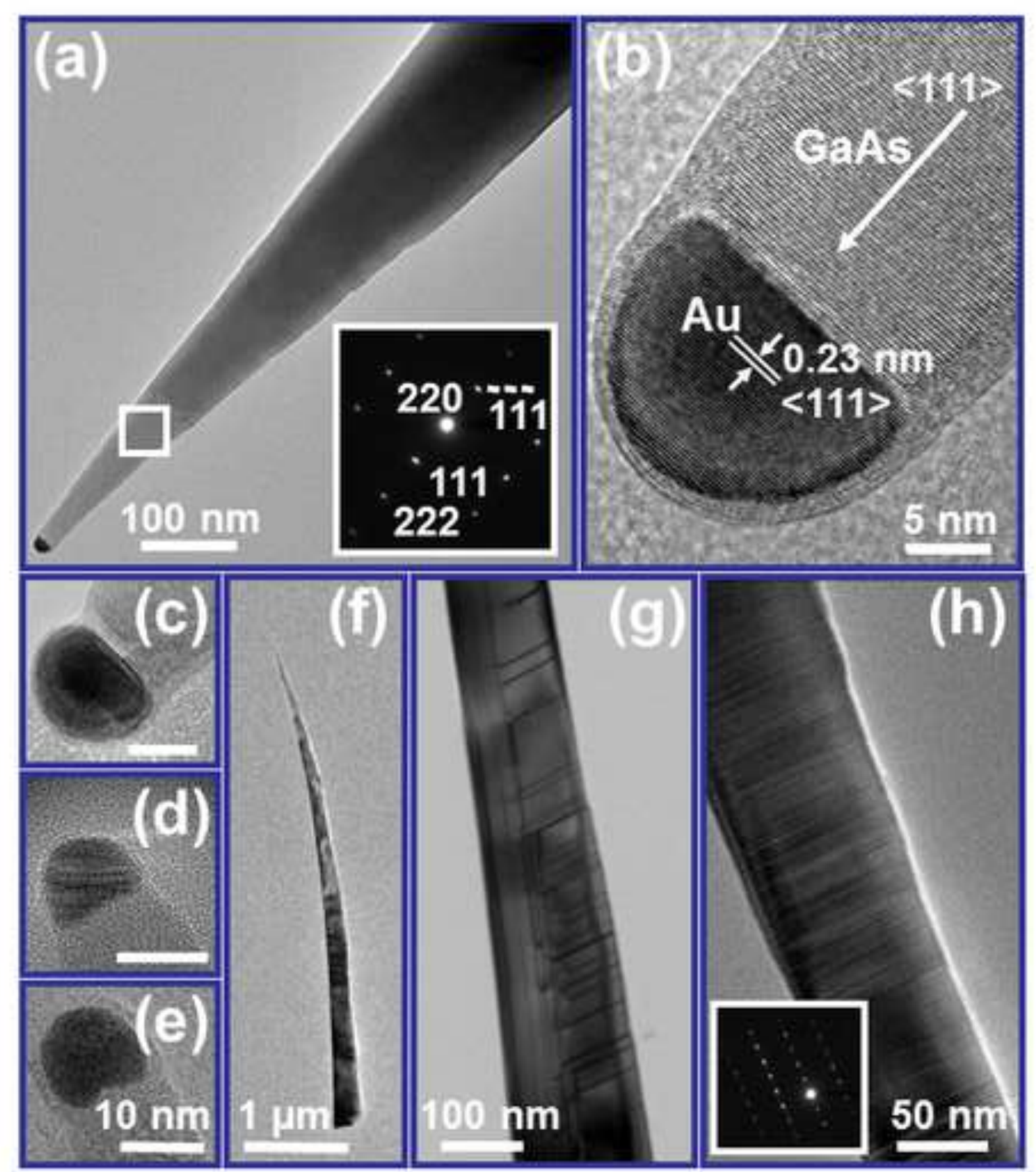



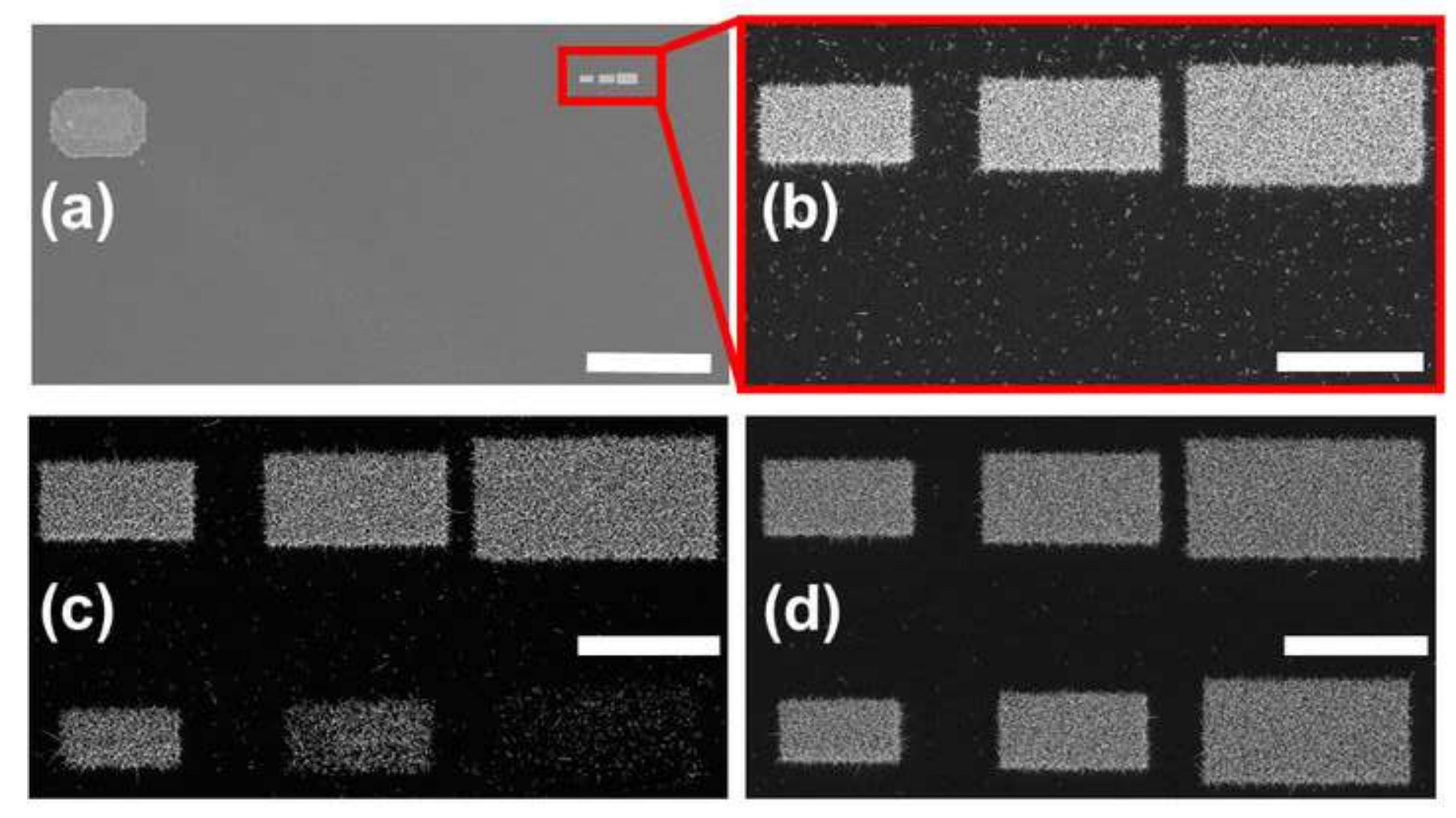


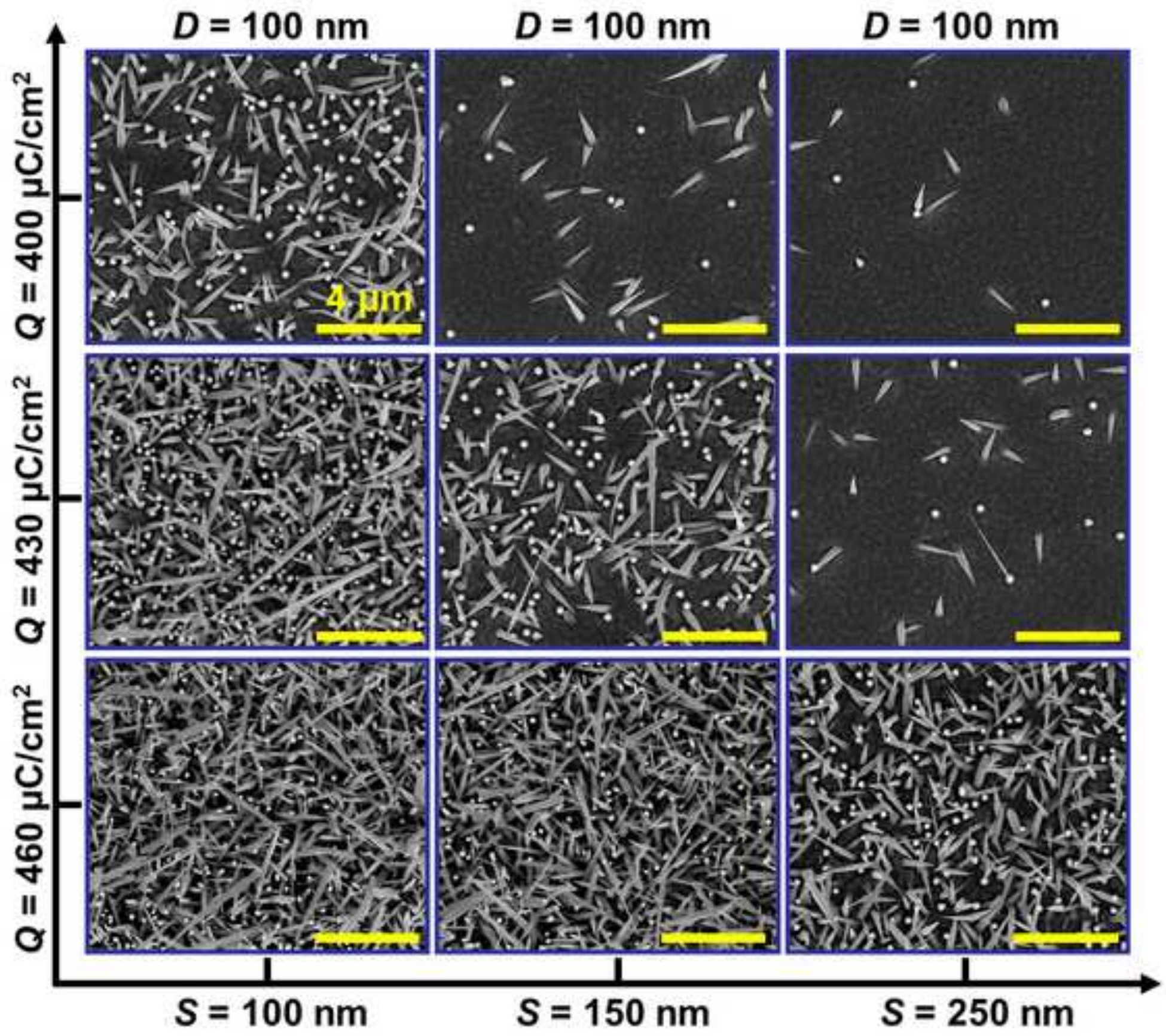


(a)

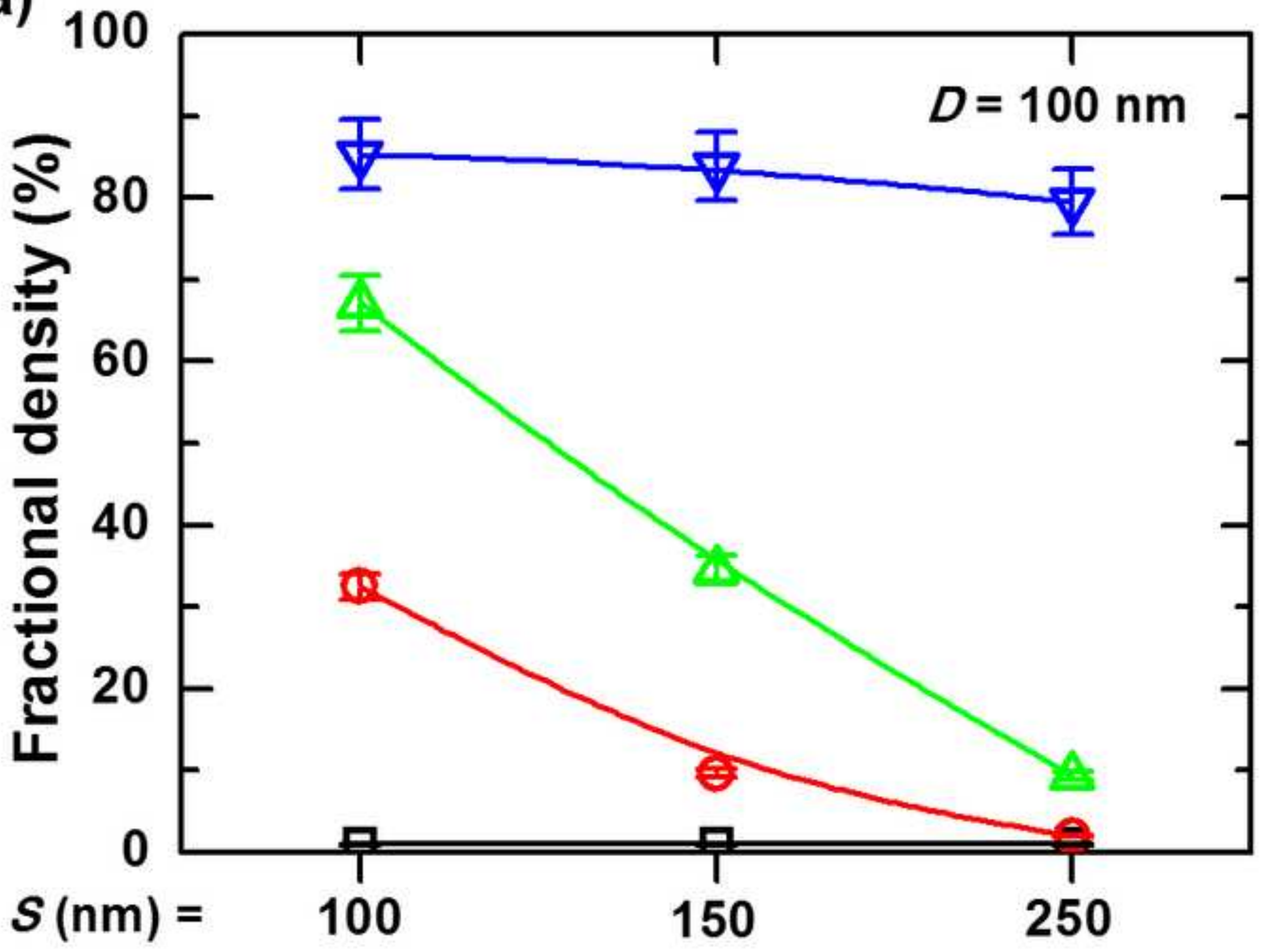


(b)

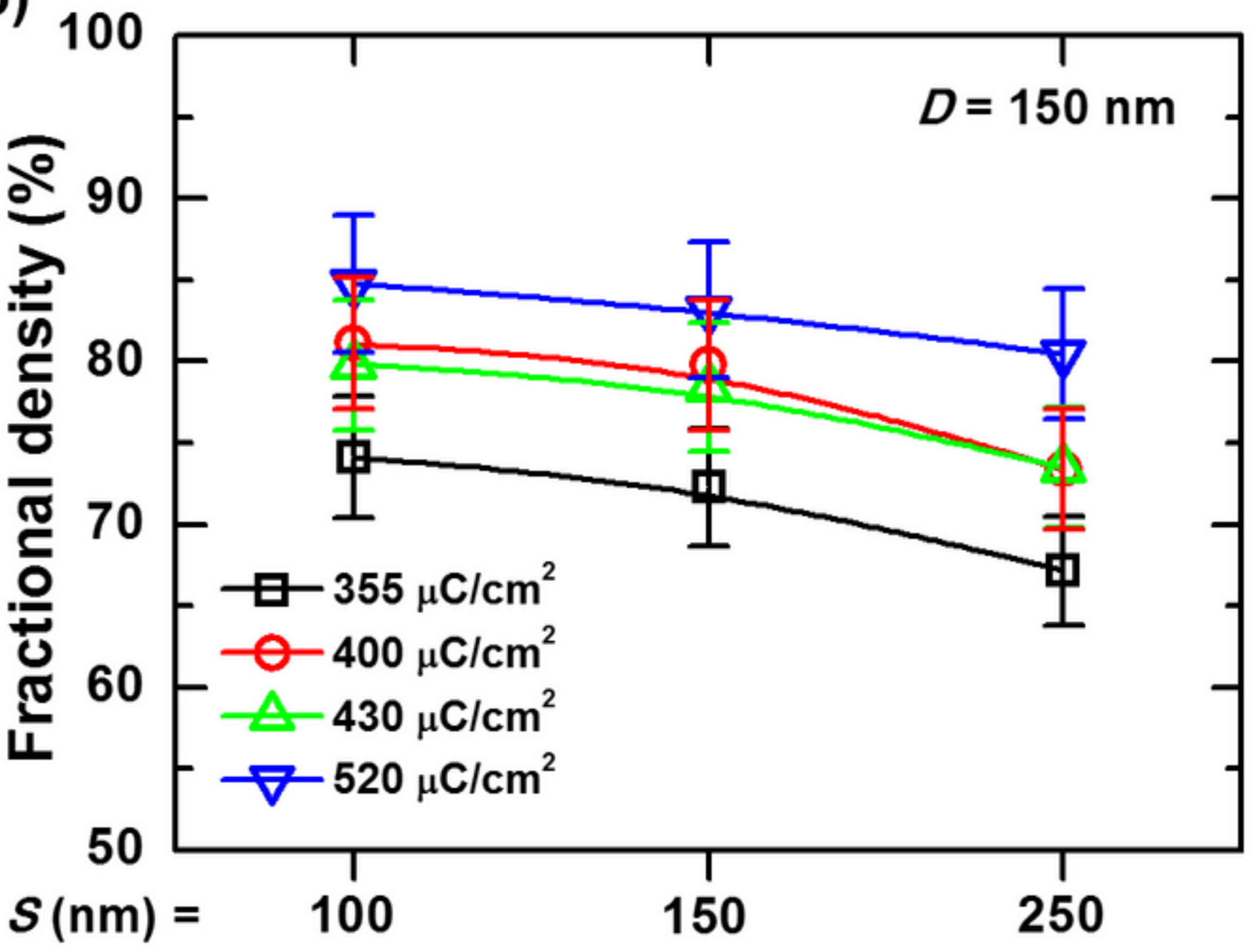

